\title{
Integrating PSP with agile process:a systematic review
}

\section{Mengjiao Shen ${ }^{1, a}$,Guoping Rong ${ }^{2, b}$ and Dong Shao ${ }^{3, c}$}

${ }^{1}$ Software Institute, Nanjing University, No.22 HankouRd,Gulou Distinct,Nanjing,Jiangsu,P.R.China.

${ }^{2}$ Software Institute, Nanjing University, No.22 HankouRd,Gulou Distinct,Nanjing,Jiangsu,P.R.China.

atracy860703@gmail.com, bronggp@gmail.com, DongShao@software.nju.edu.cn

Keywords:Agile; Software process integration; Systematic review method.

\begin{abstract}
At present, software process integration is a hot research issue which aims to improve software development. Thus, our team was organized to research this topic and proposed a cube for integration among different Classifications of processes. Nearly two years ago, we chose the integration of PSP and Agile process as our first sub-direction and showed some conclusions in [13]. During the past two years, we got more findings in the research guided by "systematic review method". As we all know that, agile process is a light-weight development process which is useful for managing unexpected changes while PSP is a typical plan-driven process which provides a disciplined way to develop software. Although they are distinct from each other, we find that PSP and agile process could complement each other.
\end{abstract}

\section{Introduction}

In modern software industry, with the increasingly fierce market competition, almost all of the software corporations are trying to find a way to produce better quality software at lower cost in a shorter time. The factors which influence achieving these three objectives in software industry can be various, specially, "Process" is considered as one of the most important factor. So far, there have been different characteristics of software development processes and these processes can promote software development to some extent. Unfortunately, still no one process can achieve the three objectives above perfectly, thus, growing number of experts are trying to improve the level of software development though "software process integration" and hope to integrate models to meet sophisticated needs. Our team was organized to research this hot topic and proposed a cube [13] for integration among different Classifications of processes. Any software development model can be mapped into a block of the cube, for this research, PSP belongs to the block that stands for process, personal and plan-driven and Agile is the opposite of the plan-driven.

In this paper, we'd like to show the entire research process under "systematic review" and the conclusions we found.

\section{Systematic Review Protocol}

In this section, we'd like to show the research process under systematic review protocol control [14]. It includes the following parts: identification of research questions, Search Strategy, inclusion and exclusion criteria, quality assessment and data extraction.

Research Question.QA1.What is the current research status of integration of PSP and agile methods? QA2.What are the main findings of these studies? QA3.How is the strength of the findings? QA4. What are the implications for research and practice?

Search Strategy. In order to collect the great literatures as many as possible, we chose eleven famous databases which we have the permission to use and the search terms is: (PSP or "personal software process") and (ASD or DSDM or FDD or XP or Lean or Xbreed or Scrum or RUP or agile or "Adaptive Software Development" or Crystal or "Dynamic Systems Development Method" or "Feature Driven Development" or "Extreme Programming" or LD or LSD or "Rational Unified Process" or EVO or "Evolutionary Project Management"). Compared to the search item in [13], we added all the process's full name to the final search item. 
Table 1.Search and Selection Results

\begin{tabular}{|l|l|l|l|l|}
\hline ID & Database Name & Rpaper & Presults & Remark \\
\hline 1 & IEEEXplore & 1 & 7 & Abstract search \\
\hline 2 & ISI Web of Science & 2 & 107 & Abstract search \\
\hline 3 & ProQuest & 2 & $<=80$ & abs:(psp) or abs:("personal software process") \\
\hline 4 & ScienceDirect & 0 & 40 & abstract, title, keyword \\
\hline 5 & SpringerLink & 2 & 14 & Abstract search \\
\hline 6 & Kluwer Online & 1 & 35 & PSP OR "personal software process" \\
\hline 7 & CiteseerX Library & 0 & 4 & Abstract search \\
\hline 8 & Wiley InterScience & 1 & 27 & Abstract search \\
\hline 9 & Google & 3 & 23 & Just title search \\
\hline 10 & ACM Digital library & 4 & 10 & Abstract search \\
\hline 11 & EBSCOhost & 0 & 15 & The same as the search item in [13] \\
\hline
\end{tabular}

Study Selection Criteria and Procedures.The inclusion and exclusion criteria were defined to access the relevant papers from the initial results of database search accurately.

Inclusion criteria:

(1) If the paper describes at least one agile method are used to promote or supplement the PSP.

(2) If the paper describes PSP is used to promote or supplement at least one concrete agile method.

(3) If the study proposes a new process model which combines the elements of PSP with the elements of Agile.

(4) If the paper expresses the attitude of suspicion or opposition to the opinion about the integration of PSP and Agile.

Exclusion criteria:

(1) Exclude Non-English literature.

(2) Exclude the papers are absolute unrelated.

After the two steps of research databases and study selection, we show the situation of accessed papers from each database in the Table 1.

Note: "Presult" means the Preliminary results of the database search; "Rpaper" equals the relevant papers we select from preliminary results according to the inclusion and exclusion criteria. Removing the duplicate papers derived from different databases, we finally get 12 papers which are listed in the References from [1] to [12].

Quality Criteria.Only High-quality literature could produce high-level conclusion of research, thus, we define ten quality criteria to assess the quality of papers. You can see these criteria in my another paper [15] and the result shows in Table 2.

Data Extraction.Data Extraction is the most crucial step to the research, thus, we design a data extraction form (Table 3) to aid this step.

Table 2.Conclusionof Quality Assessment

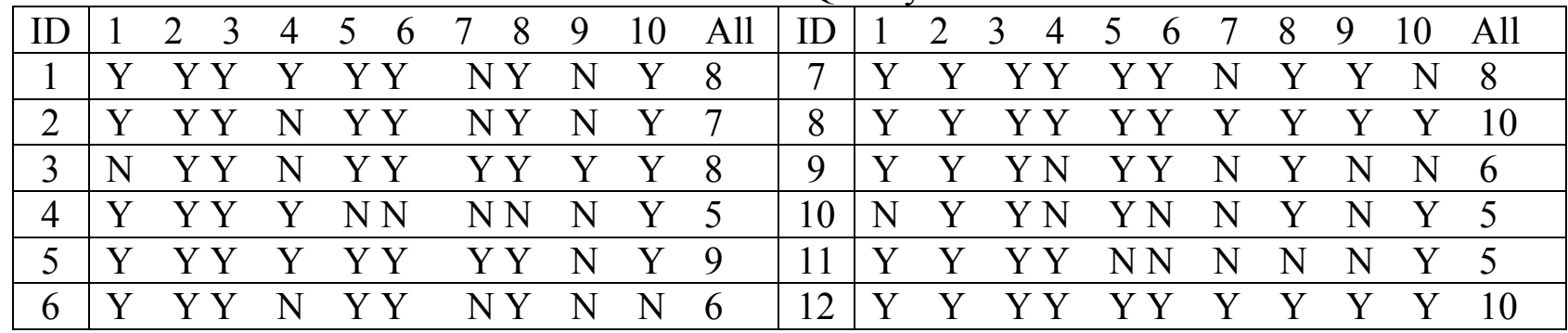

\section{Integration of PSP and Agile}

PSP and Scrum. Scrum belongs to the block that stands for process, team and agile. Scrum mainly supplies for an agile process management framework while PSP provides the skills and disciplines that are used for a technical member to estimate, plan and manage his/her work[1][11][12]. The integration of PSP and Scrum both match the needs of adaptability and predictability in modern software development [1]. 
- In detail, PSP supplement scrum:

1. Try to Break the work to a level that historical data provided by PSP is available or the size is small enough so that it can be estimated with confidence. PSP helps to make the estimation more precisely[1][12].

2. Task plan can be determined by the size estimation results and team resource estimation results. Certainly, if the schedule plan does not match the project deadline, some alternative solution may needed, to illustrate, add resource. It makes the task plan more precise [1][11][12].

3. In a quality plan, the core PSP quality indices such as phase yield, review rate, PQI and A/FR are recommended to be tracked based on quality goals. This will ensure that the product the user receives will be more error-free and increase their confidence [1].

4. PSP2.1 process which can be integrated to supplement Scrum is design template. A PSP2.1 process has eight phases, including namely planning, detailed design, detailed design review, code, code review, compile, unit test and personal postmortem. Different kinds of templates are designed to apply in different phases. To illustrate, in design review, a customized checklist is suggested [1].

5. Earned value is used to track the progress of the project [1][12]. Earned value is calculated as the percentage of total plan and a $0-100$ rule is applied to calculate the cumulative earned value. The element from PSP makes the members of project can get the information of the project status and complete dates at any time and take corrective actions soon.

- In detail, Scrum supplement PSP:

1. In a typical retrospect meeting, team members stay together to discuss the status of the project, the deviation of estimation, the quality status, the current risks and other issues. It gives an opportunity for the whole project team to identify the improvement needs[1][11][12].

2. Data collection plan defines the procedure to collect and store data such as the time spent in each phase, the size of the program and the defects injected and removed in all the phases. It standardizes the work of data collection and makes the data collected more reliable [1][11].

3. Scrum defines role and its responsibility[11][12].

We got three papers about PSP and scrum and the new methods proposed in all papers seem to be suitable for small to medium sized projects.

PSP and RUP.RUP belongs to the block that stands for process, team and agile. RUP may help to assist the software development activities by defining the efficient core process elements such as workers, activities and artifacts. As we all known that works element is the core in software development, however, RUP supports little for process improvement issues to its workers. Thus, how to quickly improve developers' skill seems to be important. PSP can be integrated to provide support to RUP's workers regarding process improvement. However, it is difficult to Incorporate PSP into RUP because PSP material should be modified so that it suits the changeable RUP context. Therefore, the lists below present how PSP at an abstract level is incorporated into RUP [6].

- In detail, PSP supplement RUP[6]:

1. PSP conducts reviews based on personal defect data and it leads to the situation that a higher a higher percentage of injected defects are probably found at the reviews. This element of PSP helps RUP to saves time and money as fewer defects are found later in the development life cycle.

2. In PSP, process data recorded in template can facilitate the software engineers to realize which areas in the software process that need improvement and opportunities for improvement of the software development process. Furthermore, the process data guides the improvement. Using this template of PSP, the software development process of RUP can be monitored, controlled and improved well.

3. Estimates done well in PSP are based on personal data. As a consequence, if the prerequisites are the same, the estimates based on personal data will be better than estimates based on data collected throughout the organization. The estimates element of PSP should be integrated into RUP to make the estimates more accurate.

4. The iterations of PSP are shorter than the iterations conducted in RUP and PSP offers support 
to plan and monitor work in short iterations. The short iteration element of PSP is integrated into RUP to let the software engineer constantly know status of the process. Besides, people could defect the deviations from the original Iteration Plan and take an appropriate action.

5. In RUP, before each new project, metrics of monitoring and controlling the software process need to be defined, however, RUP can't guarantee that its metrics is great enough to monitor and control the software process. PSP offers a set of metrics which are used to control and monitor the software development process at an individual level. Metrics element of PSP can be integrated into RUP that offers all the kind of information that a software engineer would like to have.

There is one paper we collected about PSP complement RUP and there is no any information we can found about RUP complement PSP. In our opinion, the research about integration of PSP and RUP is inadequate and more exploration needed in the future.

PSP and DSDM.DSDM belongs to the block that stands for process, team and agile. The integration of PSP and DSDM aims at small software develop companies [9].

- In detail, PSP implement DSDM [9]:

1. Unit and integration test included in PSP3 can also be integrated in within each of its development cycles.

2. The PSP suggests using proxies as a size estimating technique and RUP promotes the use of timeboxes. These proposed data collection and recording mechanisms derived from PSP make the people get the historical data from which you can determine how many LOC or Function Points (FPs) can be developed within the timebox, that is, the use of proxies will help this company refine its estimates.

3. PSP suggests DSDM do the reviews at each of the DSDM prototyping phases. For DSDM, a suggestion for improvement is that each individual developer should conduct a design and code review while another technical peer does the testing. It ensures that "clean" versions of software are used as input to the next increment. Also, a technical peer charges for testing can save developers'confidence.

4. Some documentation provided by the PSP could be included in a quality plan of RUP, for example, process script, design and code checklist and RIP. The PIP provides a way to document process disadvantages and suggested solutions. PIP copies which could be used to assist in developing and implementing the quality plan should be retained in the quality plan.

5. At present, the DSDM consortium recommends no specific approach towards collecting metrics such as defect metrics, productivity metrics, size metrics, time/schedule metrics, extended metrics and maintenance metrics. All of these metrics are well defined in PSP. Developers can decide which metrics should be applied according the real situation.

We just found one paper about integration of PSP and DSDM and the research is really not enough. Meanwhile, there is still no opposing viewpoints emerged [9].

PSP and XP.XP belongs to the block that stands for practice, team and agile. XP helps to deal with the situation of developing projects with often changing requirements and high quality demands in small team while PSP helps developers as individuals to understand and improve their personal performance. XP contains twelve practices: The Planning Process, Short Releases, Metaphor, Simple Design, Test first, Refactoring, Pair Programming, Collective Ownership, Continuous Integration, 40-hour Week, On-site Customer, and Coding Standard.

- In detail, PSP implement XP

1. PSP provides script to XP for supporting each engineering activity and facilitating its correct completion. In different cases, we should appropriately choose the scripts being followed and the data we need to be filled in the forms [7][2].

2. PSP guides XP to estimate and plan work. The estimate work could be made according to the actual completion statistics from past projects and also with some tools and methods $[8][6][5][2]$.

3. Compared to XP, PSP seems to be more structured. XP lacks of detail development processes and PSP could supply various kinds of framework constitutes of several processes 
[2].

4. PSP can supplies measures element which XP lacks. Measures helps to optimize the time and cost planning [2][12].

5. PSP supplements XP that Recording and analyzing defects. The recorded defects can be analyzed to avoid making the error again or solve the same defect in other future project. Besides, it increases the product's quality [5][10].

6. Earned value analysis of PSP is used to track progress and makes sure that the project is proceeding on schedule [8][10].

7. PSP is organized in levels, and companies could choose a level according their current state and maturity level, and gradually improve them [2].

- In detail, XP implement PSP

1. Engineers perform a CRC card role play and use case analysis as input to a thorough (UML) high-level class diagram. These could be added into PSP to get requirement [8].

2. Because pair programming is essentially a continuous review, the design review and code review procedures of PSP are changed as iterative pair-reviews. The checklist used in review should be designed into two forms (one is for single and another for pairs). With pair programming, problem identification occurs on a minute-by-minute basis. These continual reviews not only outperform formal reviews in their defect removal speed, but they also eliminate the programmer's distaste for reviews [8][10].

3. One practice of XP is On-site Customer that means active participation of the client who could express his requirement for the developed software at any time [10].It saves time for realizing features which are not needed.

4. One practice of XP is test-driven development that means test first before begin to develop the software. This practice can be used to in the testing of PSP [3][10][12]. It makes a faster software development and increased quality.

5. Continuous integration is another practice which can implement PSP. Continuous integration constitutes of the sub-practices of source control, versioning, automated builds, automated test executions, and automated defect submitting [3][10].

This keeps all the programmers on the same page, and speeds the development progress.

6. The practice of simple design of XP should be used into PSP to meet the changeable requirements and contributes to its faster development. [3][10].

We found eight papers about integration of PSP and XP and got many conclusions. However, only one of three research conclusion was verified in the real project. Thus, the conclusions should be verified in the real projects and more researches are need in the future.

Benefits of Combining PSP with Agile. In the paper[13], it mainly concludes six points about the benefits of using PSP to complement the Agile. These six benefits are listed as follows:

1. PSP offers metrics and data collecting mechanisms.

2. PSP supports to make more reasonable estimation.

3. PSP helps to optimize plans.

4. PSP offers support to produce software products up to quality goals.

5. PSP provides additional documentation for agile methods.

6. PSP helps individual engineers to improve their personal performance gradually.

In this part, we'd like to add one more point that PSP helps the Agile to monitor and track the software process. It is the earned value that assist the team members to track the project progress and know the status of the project [1][8][10][12].

\section{Conclusion}

In this paper, we research the integration of PSP and Agile software development method which includes ASD, DSDM, FDD, XP, Lean, Scrum, RUP, Crystal, EVO and other agile methods. However, in the existed papers, the agile processes we found to be integrated to PSP are only XP, DSDM, RUP and Scrum. We summed up the situation about these four integration individually and 
also the evidences that PSP implements the agile software development. Besides, remember that when incorporate PSP into agile methods, the PSP process should be adapted to the project's own needs and applied accordingly. What's more, software tools for assistance should also be used to improve efficiency.

The future work is to investigate other software process integration under the same research process and our final aim is to fill the cube.

\section{Table 3.Data Extraction Form}

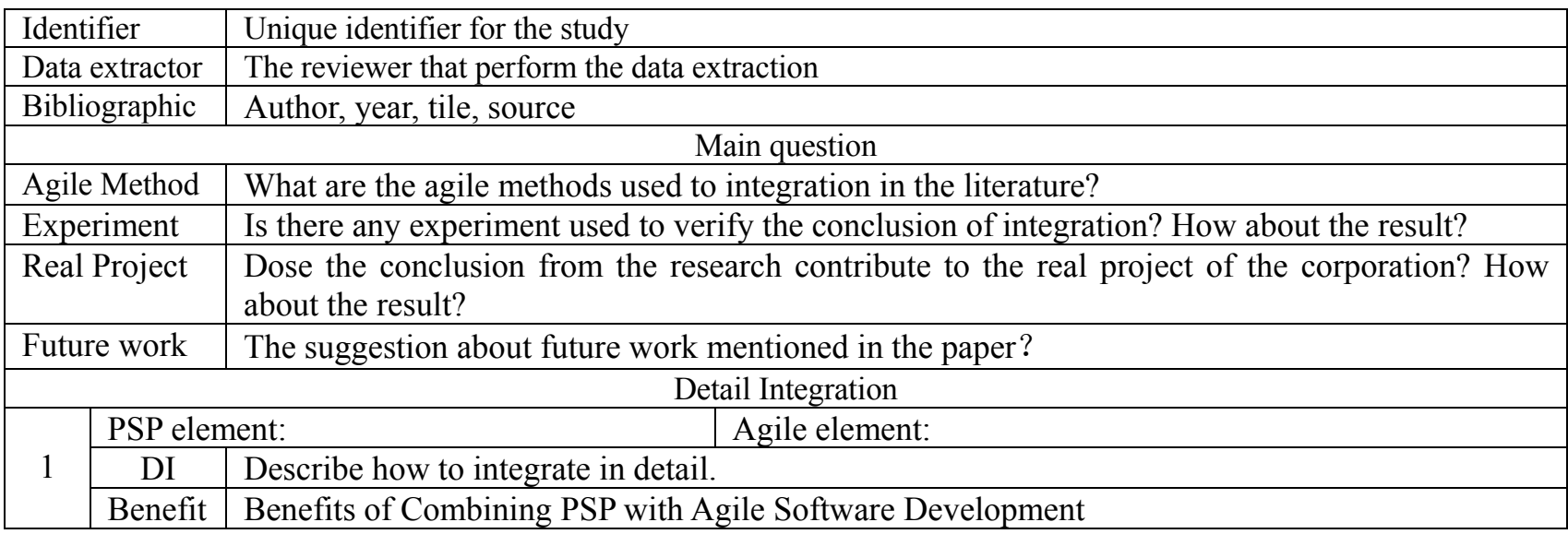

\section{References}

[1] GuopingRong, Dong Shao and HeZhang, SCRUM-PSP: Embracing Process Agility and Discipline, Software Engineering Conference (APSEC), 2010 17th Asia Pacific.

[2] IlianMihaylov, The expert approach - a case study, CompSysTech '03 Proceedings of the 4th international conference conference on Computer systems and technologies: e-Learning.

[3] DzhurovYani, Personal Extreme Programming - An Agile Process for AutonomousDevelopers.In Proceedings of International Conference on Software, Services \& Semantic Technologies, October 28-29, 2009, Sofia, Bulgaria.

[4]Agarwal, R. and Umphress, D.2008. Extreme Programming for a Single Person Team. In Proceedings of the 46th Annual Southeast Regional Conference on XX,ACM-SE 08, March 28-29,2008,Auburn,AL,USA, 82-87.

[5] TeodoraBozheva,Practical Aspects of XP Practices,Computer Science, 2003, Volume 2675/2003, 1011, DOI: 10.1007/3-540-44870-5_52.

[6] Svensson, Harald, Developing Support for Agile and Plan-Driven Methods, KTH, Sweden.

[7] Ilieva, S. and Stefanova,E .2002.Expert approach for e-business software development. In Proceedings of International conference Basic Technologies for E-business, 2002, Albena, 16-18 September.

[8] Williams, L., Integrating Pair Programming into a Software Development Process, Software Engineering Education and Training,2001. Proceedings.14th Conference on.

[9] Coleman, G. and Verbruggen, R.1998. A quality software process for rapid application development. Software Quality Journal 117, (1998), 107-117.

[10] Eliza Stefanova, Sylvia Ilieva,Expertapproach and framework foranalyzing experiments with it,Proceedings of 16th International Conference on Systems for Automation of Engineering and Research (SAER'2002).

[11] SuphakSuwanya and WerasakKurutach, Applying Agility Frameworkin Small and Medium Enterprises.

[12] DeysianeSande, A Strategy to Support Software Planning Based on Piece of Work and Agile Paradigm.

[13] Wengrong Yang and MengjiaoShen, Investigating the Benefits of Combining PSP with Agile Software Development, EAST2011. 
[14] Barbara Kitchenham,Procedures for Performing Systematic Reviews, Empirical Software Engineering National ICT Australia Ltd. Bay 15 Locomotive Workshop Australian Technology Park

[15] Mengjiao Shen, Wenrong Yang, Applying Agile Methods to Embedded Software Development: A Systematic Review, SEES 2012 of ICSE. 